\title{
Magnesium degradation observed in situ under flow by synchrotron radiation based microtomography
}

\author{
Frank Feyerabend ${ }^{\mathrm{a}}{ }^{1}$, Thomas Dose ${ }^{\mathrm{a} *}$, Yuling $\mathrm{Xu}^{\mathrm{a}}$, Felix Beckmann ${ }^{\mathrm{a} 2}$, Michael Stekker ${ }^{\mathrm{b}}$, Regine \\ Willumeit-Römer ${ }^{\mathrm{a}}$, Andreas Schreyer ${ }^{\mathrm{a}, \mathrm{c}}$, Fabian Wilde ${ }^{\mathrm{a}}$, Jörg U. Hammel ${ }^{\mathrm{a} 3}$ \\ ${ }^{a}$ Helmholtz-Zentrum Geesthacht, Institute of Materials Research, Max-Planck-Str. 1, D-21502 \\ Geesthacht, Germany; ${ }^{b}$ MeKo Laser Material Processing, Im Kirchenfelde 12-14, D-31157 Sarstedt, \\ Germany, ${ }^{\mathrm{E} E u r o p e a n}$ Spallation Source (ESS), Tunavägen 24, 22363 Lund, Sweden
}

\begin{abstract}
The use of degradable magnesium based implants is becoming clinically relevant, e.g. for the use as bone screws. Still there is a lack of analyzing techniques to characterize the in vitro degradation behavior of implant prototypes. The aim of this study was to design an in situ environment to continuously monitor the degradation processes under physiological conditions by time-lapse SR $\mu \mathrm{CT}$. The use of physiological conditions was chosen to get a better approach to the in vivo situation, as it could be shown by many studies, that these conditions change on the one hand the degradation rate and on the other hand also the formed degradation products. The resulting in situ environment contains a closed bioreactor system to control and monitor the relevant parameters $\left(37^{\circ} \mathrm{C}, 5 \% \mathrm{O}_{2}, 20 \% \mathrm{CO}_{2}\right)$ and to grant sterility of the setup. A flow cell was designed and manufactured from polyether etherketone (PEEK), which was chosen because of the good mechanical properties, high thermal and chemical resistance and radiographic translucency. Sterilization of the system including the sample was reached by a transient flush with $70 \%$ ethanol and subsequent replacement by physiological medium (Modified Eagle Medium alpha). As proof of principle it could be shown that the system remained sterile during a beamtime of several days and that the continuous SR $\mu \mathrm{CT}$ imaging was feasible.
\end{abstract}

Keywords: SR $\mu \mathrm{CT}$, tomography, magnesium, in situ, sample environment, degradation, corrosion

\section{INTRODUCTION}

Research on magnesium and its alloys as biodegradable implant materials in vitro is hampered by various challenges: (I) the lack of standardizations concerning sample geometries and characterization methods as well as the need to extend available normatives to these sample classes ${ }^{1-3}$; (II) the variety of methods for the determination of degradation rates (e.g. immersion tests, electrochemical methods, weight loss measurements etc.), and (III) the missing correlation between in vitro and in vivo results ${ }^{4}$, which can be approximated by the choice of solutions and the application of physiological conditions ${ }^{5,6}$. Therefore momentarily only in vivo experiments are giving "real" values, although also the site of implantation and the used animal model will render different values ${ }^{7}$. In recent years the methodology to perform in vitro methods has been greatly improved including immersion under cell culture conditions (i.e. $37^{\circ} \mathrm{C}, 5 \% \mathrm{CO}_{2}, 21 \%$ $\mathrm{O}_{2}, 95 \%$ relative humidity and in cell culture medium). It could be shown that in cell culture environment degradation rates more similar to those observed in vivo could be obtained ${ }^{8}$. Moreover, in this artificial environment additional chemical reactions are taking place, which lead to a very complex degradation environment, also more comparable to the findings in vivo ${ }^{5,6,9-11}$.

Laboratory based and in vivo micro-computed tomography instruments $(\mu \mathrm{CT})$ are used as a standard technique to follow the degradation behavior in vivo e.g. ${ }^{12,13-15}$. Furthermore, several attempts were undertaken to analyze ex vivo samples by synchrotron radiation based microtomography ${ }^{16-18}$. However, these measurements are only end-point determinations and do not reveal the dynamics of the complex degradation behavior of magnesium materials.

It is generally agreed that microtomography is a valid technique for determining degradation rates. The advantage of this technique is that it is non-destructive, giving room for further analysis of the sample, for example by histology ${ }^{19,20}$. The 3D-volume of the sample can be analyzed with high density and spatial resolution. Compared to laboratory X-ray sources, monochromatic X-ray sources at synchrotrons (SR $\mu \mathrm{CT}$ ) can be used to achieve better density resolution in absorption contrast tomography ${ }^{14,16,21}$ as well as much higher scan speeds. Since the absorption coefficient is proportional to the density of a material and to the cube of atomic number, this allows for distinguish areas with different

Developments in X-Ray Tomography X, edited by Stuart R. Stock, Bert Müller, Ge Wang, Proc. of SPIE Vol. 9967, 99671X · ( 2016 SPIE · CCC code: 0277-786X/16/\$18 · doi: 10.1117/12.2241085 
effective atomic numbers. It is therefore the first choice for in situ measurements, although it is not easily accessible. Until now, it is restricted to end point measurements, i.e. after the immersion of the samples ${ }^{22}$. However, to shed a light on the degradation process itself it would be favorable to have a time resolved measurement. This could be obtained by repeated measurement and immersion cycles, but this approach is impractical, because it is time-consuming and may also lead to a change in the degradation behavior. The aim of our study therefore was to design an in situ chamber for synchrotron radiation-based time-lapse X-ray microtomography (SR $\mu \mathrm{CT}$ ) with the following specifications: (I) the same conditions as used in cell culture environment should be obtainable; (II) an adjustable continuous flow for medium exchange should be established; (III) the system should be sterilizable and able to maintain sterile conditions during the experiment and (IV) the inclusion of different sensors for online monitoring of $\mathrm{pH}, \mathrm{CO}_{2}$ and $\mathrm{O}_{2}$ should be possible.

\section{MATERIALS AND METHODS}

\subsection{Beamlines}

Experiments were performed at the storage ring DORIS III at the Deutsches Elektronen-Synchrotron DESY ${ }^{23}$. The tomography endstations of the beamlines BW2 and W2 were used, both operated by the Helmholtz-Zentrum Geesthacht $\mathrm{HZG}^{24}$. Tomograms at the BW2 beamline were taken using a photon energy of $18 \mathrm{keV}$, a field of view of $2.0 \mathrm{~mm} \mathrm{x}$ $1.3 \mathrm{~mm}$ and a spatial resolution of $4.45 \mu \mathrm{m}$ at a voxel size of $2.6 \mu \mathrm{m}$ in the reconstructed volume. At the endstation W2 an energy of $23 \mathrm{keV}$ was used with a field of view of $6.8 \mathrm{~mm}$ x $6.8 \mathrm{~mm}$ and a spatial resolution of $5.8 \mu \mathrm{m}$ and a voxel size of $4.4 \mu \mathrm{m}$ in the reconstructed volume. Both beamlines provided a hanging rotation stage which had to be taken into account for the design of the in situ corrosion cell. At the beamline BW2 scan duration for one tomogram was between 160 to 340 minutes, whereas at beamline W2 faster scans (between 150 to 180 minutes) were possible.

\subsection{In situ corrosion cell}

For the design of the sample cell and its integration into the beamline instrument the following boundary conditions had to be taken into account: (I) the sample should be immersed in fluid all the time, (II) sterility should be granted, (III) the amount of the material other than the sample in the field of view (FOV) should be minimal, and (IV) during microtomography reference images (flat field) have to be recorded, which require a horizontal movement of the sample out of the FOV within the corrosion cell. Due to its good mechanical properties, high thermal and chemical resistance and radiographic translucency the chamber was manufactured from polyether ether ketone (PEEK, Arthur Krüger, Barsbüttel, Germany). Continuous sample immersion with a constant flow rate was obtained by placing an overflow edge in the chamber (Figure 1). Medium inlet was placed below the outlet. To reach sterile conditions a continuous flow of argon has been established above the medium surface. A window for the x-ray beam had a reduced material thickness of $500 \mu \mathrm{m}$ on both sides of the chamber. Horizontal movement of the rotation axis for reference images was established through a slit opening in the cover (made from stainless steel) and introducing a seal/gasket made from nitrile. Additional $\mathrm{CO}_{2}$ and $\mathrm{O}_{2}$-sensors (PreSens $\mathrm{GmbH}$ ) were inserted in the measuring chamber.
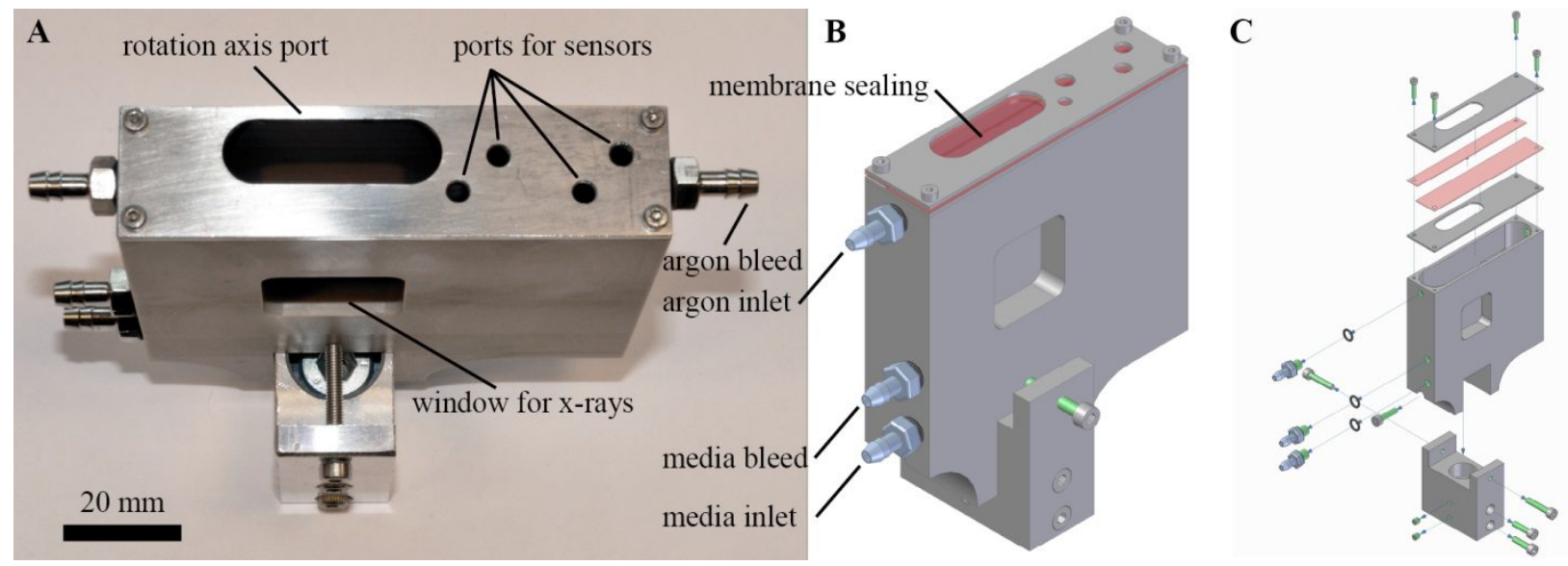

Figure 1: A) Photograph and schematic drawing B-C) of the in situ corrosion chamber system for X-ray tomography at synchrotron radiation facilities. 


\subsection{Experimental setup}

Besides the in situ chamber a bioreactor system (Vario 1000; Medorex e.k., Nörten-Hardenberg, Germany) was used to maintain a continuous cell medium flow through the chamber at a flow rate of $1 \mathrm{ml} / \mathrm{min}$. Oxygen concentration, $\mathrm{pH}$ (by $\mathrm{CO}_{2}$-addition), temperature and flow rate were measured and regulated using a peristaltic pump (TL/15EAD, Medorex e.k) and the related bioreactor control system. Sterilization was done by a transient flush of the system with $70 \%$ ethanol (Merck KgaA, Darmstadt, Germany) for $1 \mathrm{~h}$ prior to the experiment, with the sample already inserted. Afterwards ethanol was replaced by $250 \mathrm{ml}$ Modified Eagle Medium alpha ( $\alpha$-MEM, Life Technologies, Darmstadt, Germany) with $10 \%$ fetal bovine serum (FBS, PAA Laboratories, Linz, Austria).

\subsection{Sample preparation}

Mg-10Gd-1Nd (10 \% gadolinium, $1 \%$ neodymium) samples were produced as tubes. Metallic Mg (99,94 \%) was molten in a mild steel crucible under a protective atmosphere ( $\left.\mathrm{Ar}+2 \% \mathrm{SF}_{6}\right)$. Pure Gd and $\mathrm{Nd}(99,9 \%)$ were added at a melt temperature of $720^{\circ} \mathrm{C}$, after which the melt was stirred for $30 \mathrm{~min}$ at $200 \mathrm{rev} \mathrm{min}^{-1}{ }^{25}$. Permanent mould direct chill casting was used to prepare the ingot. The size of the ingot was $20 \times 12 \times 6 \mathrm{~cm}$. The ingot was extruded to a rod with a final diameter of $6 \mathrm{~mm}$ (Strangpreßzentrum Berlin, Berlin, Germany). This rod was further processed to a tube with 15 $\mathrm{mm}$ length, $1 \mathrm{~mm}$ diameter and a wall thickness of $100 \mu \mathrm{m}$ by tube drawing (G. Rau GmbH, Pforzheim). Sterilization of the final tubes was done by gamma-irradiation ( $29 \mathrm{kGy}$, In Core Irradiation facility, Helmholtz-Zentrum Geesthacht).

\subsection{Metallographic preparation}

Specimen were ground using silicon carbide emery paper up to 2500 grit, polished with $1 \mu \mathrm{m}$ colloidal silica (OPS) and cleaned using ethanol. Etching was performed in a solution containing $8 \mathrm{~g}$ picric acid, $5 \mathrm{~mL}$ acetic acid, $10 \mathrm{~mL}$ distilled water and $100 \mathrm{~mL}$ ethanol. Microstructure was characterized using light microscopy (Reichert-Jung MeF3) with a digital camera. A Zeiss Ultra 55 (Carl Zeiss GmbH, Oberkochen, Germany) scanning electron microscope (SEM) equipped with energy dispersive X-ray (EDX) analyzer was used to observe the microstructure at $20 \mathrm{KeV}$.

\subsection{Image processing and quantitative analysis}

For tomographic reconstruction of the 3D datasets, the algorithm "back projection of filtered projections" ${ }^{26}$ was used to yield 32-bit floating-point image-stacks with isotropic voxel size. Segmentation of degradation layers was performed by thresholding in Image ${ }^{27}$. Further image analysis, e.g. edge filtering, histogram analysis and volume measurements to characterize the degradation layers, the location of the corrosion front and volume fractions of bulk material and degradation products were performed in ImageJ as well.

\section{RESULTS}

Setup of the bioreactor system

A closed bioreactor system coupled to an in situ corrosion cell was installed and successfully operated in the beamlines BW2 and W2 at the storage ring DORIS III at DESY. A series of measurements documenting Mg degradation was

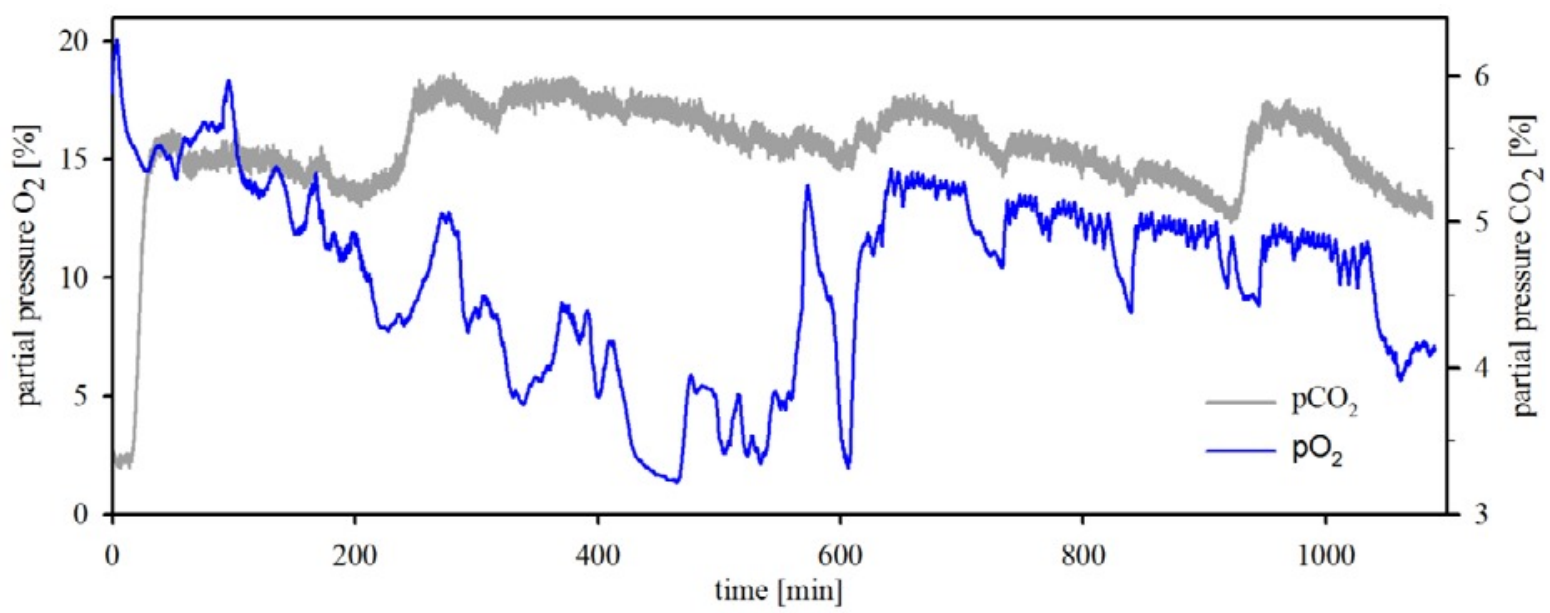

Figure 2: Partial pressure of oxygen (blue curve) and carbon dioxide (grey curve) in the in situ chamber during the beamtime. 

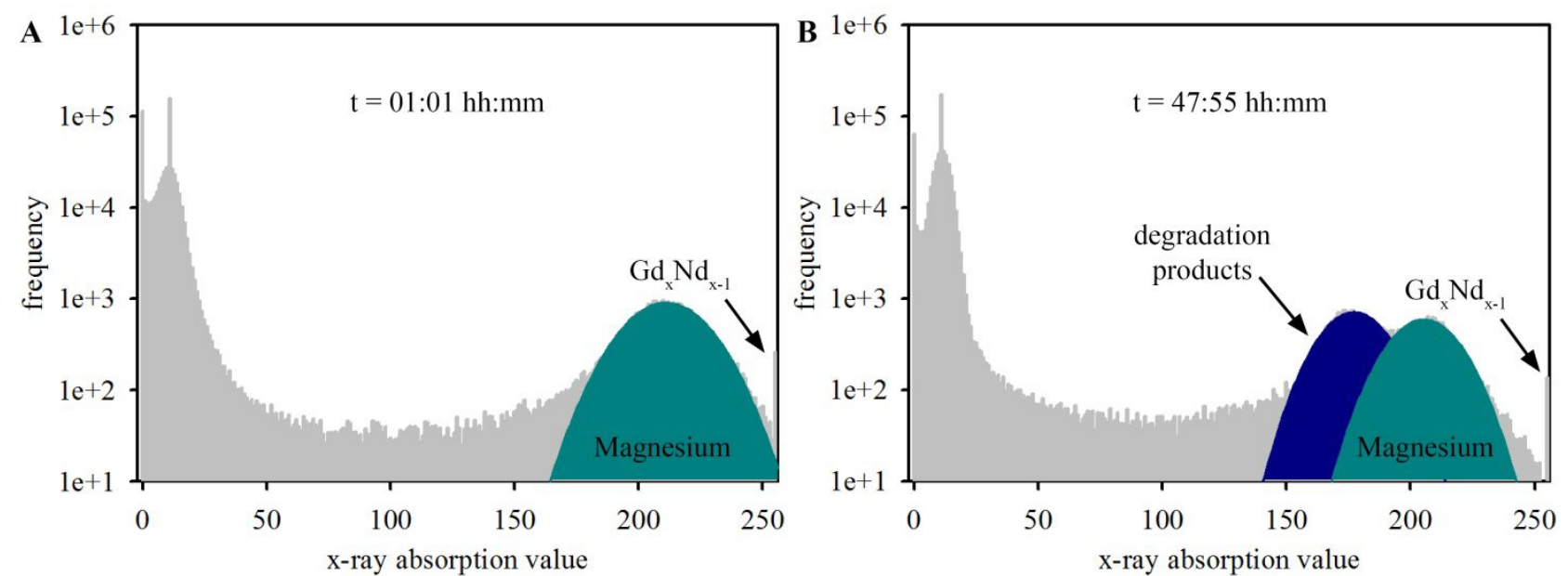

Figure 3: Histograms of different time points and Gaussian peak fits for bulk material and degradation products. A) Histogram from the first time point including already some degradation products, B) histogram at the end of the measurement series.

recorded during a consecutive 5 days beamtime. Sterility of the medium could not be granted over the entire experiment period, as indicated by high fluctuations in the amount of oxygen consumption (Figure 2). However, regulation of $\mathrm{CO}_{2}$ as buffering gas was constantly achieved around $5 \%$ (Figure 2). Although temperature in the bioreactor was constantly regulated at $37^{\circ} \mathrm{C}$, the temperature in the in situ cell was much lower $\left(24^{\circ} \mathrm{C}\right)$.

\section{Qualitative analysis of degradation}

An analysis of the histograms over the interval of the time series revealed a change in composition of bulk material and degradation products. It was possible to distinctly discriminate the low absorbing hydrogen, water, magnesium and the alloyed elements ( $\mathrm{Gd}$ and $\mathrm{Nd}$ ) (Figure 3). Comparing the histograms from early and late time points a new peak appeared in later ones which corresponds to newly formed degradation products (Figure 3). On the basis of the measured

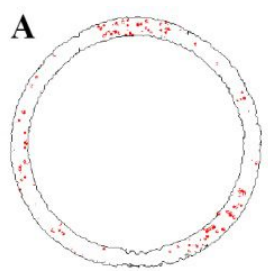

01:01 hh:mm

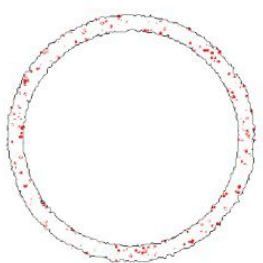

22:38 hh:mm

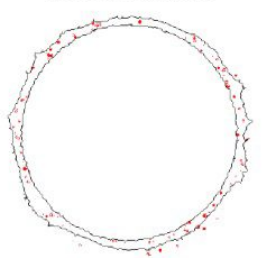

42:16 hh:mm

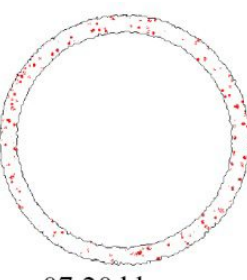

07:20 hh:mm

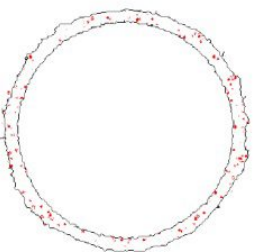

29:43 hh:mm

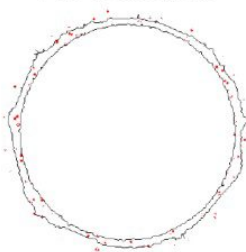

47:55 hh:mm

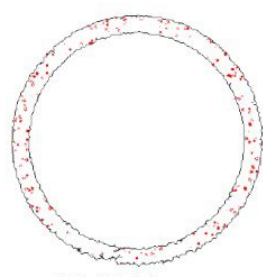

16:09 hh:mm

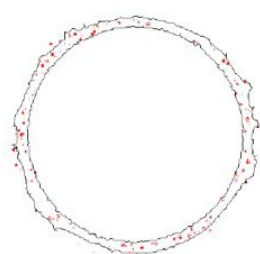

35:35 hh:mm

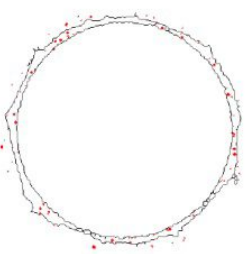

53:34 hh:mm

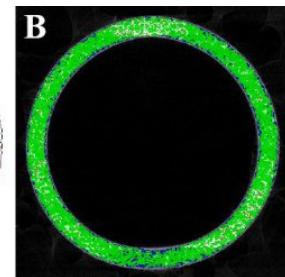

01:01 hh:mm

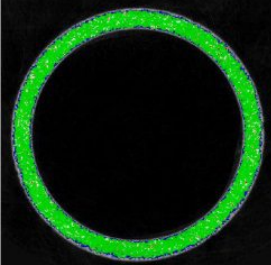

22:38 hh:mm

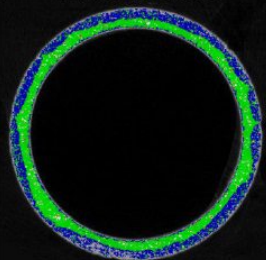

42:16 hh:mm
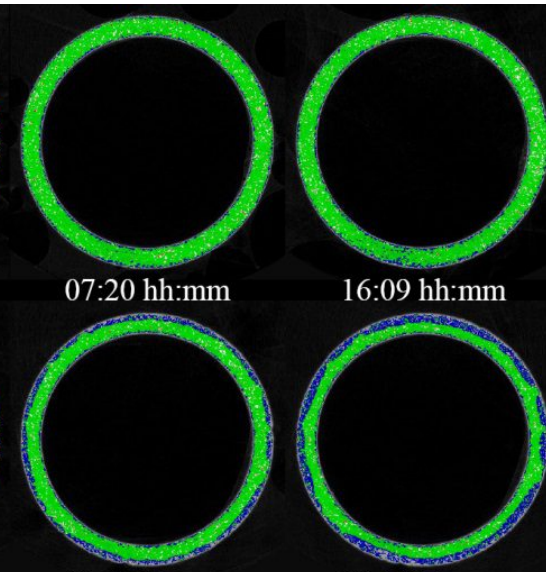

29:43 hh:mm

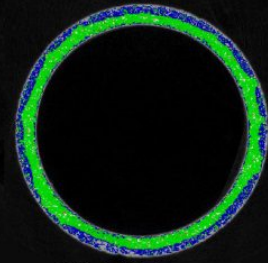

35:35 hh:mm

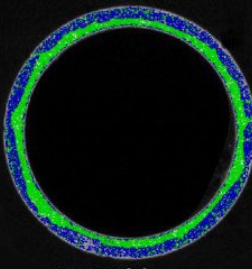

47:55 hh:mm

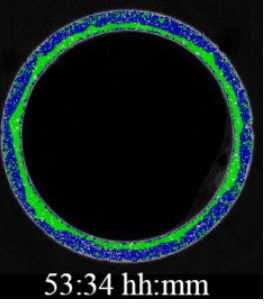

Figure 4: Timelapse image sequence of a selected horizontal slice from the 3D volume dataset. A) Visualization of the corrosion front and segregation products. B) Degradation layer (blue) and bulk material (green). Sample diameter: 1.6 mm 


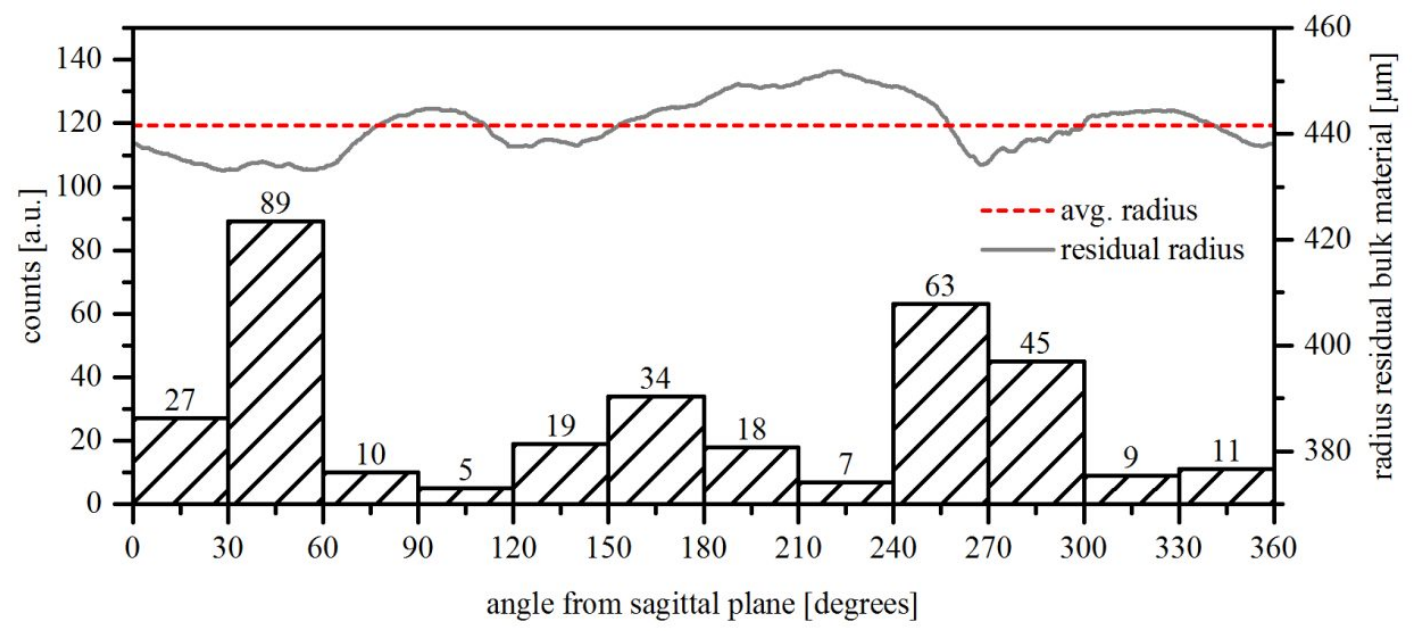

Figure 6: Degradation front vs. number of segregated gadolinium particles after 01:01 hh:mm incubation in the media.

absorption it can be stated, that the optical density of the degradation products is lower than that of magnesium. Based on the recorded tomographic image stacks it was possible to qualitatively visualize the degradation front, as well as the change in general morphology over time (Figure 4). Overall the general appearance of the sample did not change during the time series of measurements, whereas the degradation front moved throughout the tube wall. This indicates a replacement of the bulk material by degradation products. Due to a trapped air bubble inside the tube the corrosive fluid had no contact to the sample inner surfaces. Therefore, corrosion of the sample started only from the outer surface.

An analysis of the position of the Gd/Nd-segregations revealed a high position stability of the segregations compared to their initial position (Figure 5). In the location of high accumulation of $\mathrm{Gd} / \mathrm{Nd}$-segregations also the degradation of the
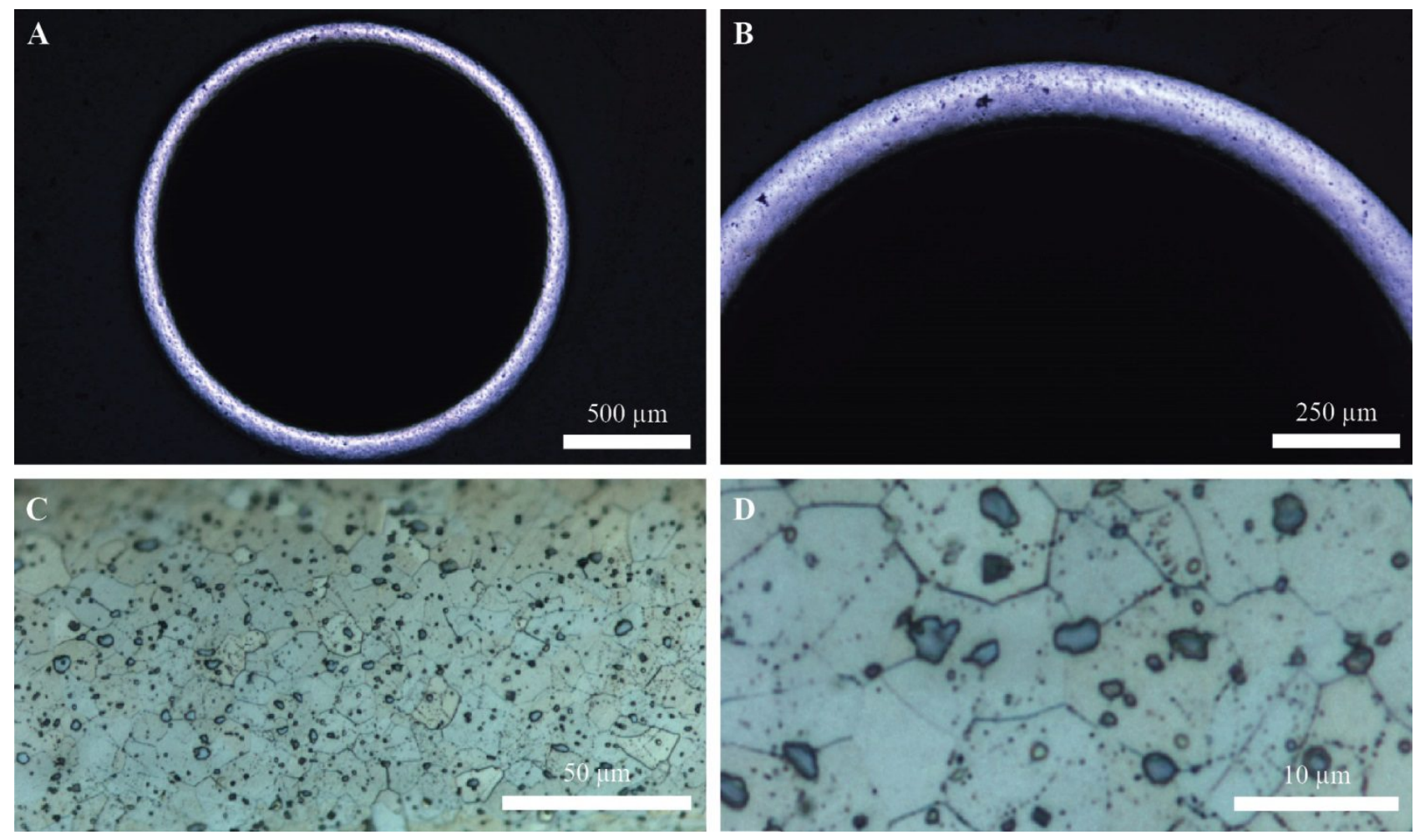

Figure 5: Micrographs of the microstructure of the Mg-10Gd-1Nd tube with increasing magnification. 

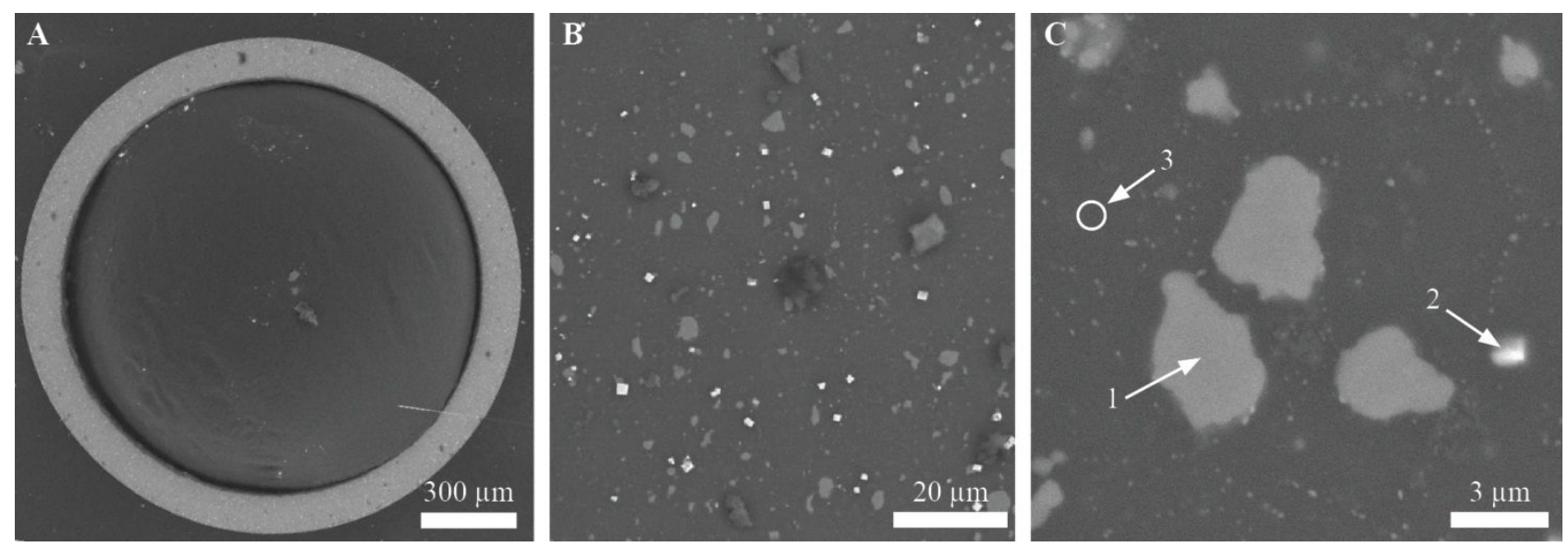

Figure 7: EDX-analysis of Gd and Nd distribution in the alloy. A) Overview of the sample. B) and C) increasing magnifications and sample locations for the EDX analysis. Areas for the EDX measurement $(1,2,3)$ are highlighted in C).

bulk material is higher compared to the average degradation for the respective image slice (Figure 5). Based on metallographic preparations an analysis of the actual microstructure was performed. Micrographs of Mg-Gd-Nd alloy are shown in Figure 6. It reveals that the alloy grain size is around $10 \mu \mathrm{m}$. Large amount of intermetallic phases are distributed in the alloy matrix. This is similar to the segregation observed in the tomographic analysis. From the microstructure analysis it is obvious that Gd and Nd distributions vary in different zones. For their identification, EDX analysis was performed on these zones (Figure 7, Table 1). Grey particles, like position 1, are frequently found, the EDX analysis of this particle indicates that it is composed of Mg (97.1 at.\%), Nd (2.4 at.\%) and Gd (0.5 at.\%). The white particle (Position 2), having a very regular blocky shape, is chosen as an example, which is extremely rich in Gd (6.0 at.\%) and additionally contains $\mathrm{Mg}$ (93.7 at.\%) and $\mathrm{Nd}$ (0.2 at.\%). In the Mg matrix, like position 3, there are little Gd (1.4 at.\%) and $\mathrm{Nd}$ (0.1 at.\%) elements.

Edge filtering allowed for an automated identification of the border between degradation products and bulk material. A transformation of the border into polar coordinates enabled the calculation of the mean residual radius which served as a basis to calculate degradation speed. We have identified a depth of penetration of $11.5 \pm 1.8 \mathrm{~mm} / \mathrm{a}$ for the investigated Mg-10Gd-1Nd alloy.

\section{DISCUSSION}

Magnesium degradation is a highly dynamic and complex process, especially when physiological conditions are applied ${ }^{28,29}$. It is highly dependent on the choice of appropriate solutions ${ }^{30}$ and on the applied environment ${ }^{6,}{ }^{9}$, however, just for determining the initial degradation there are nearly no direct methods available. Therefore, our approach focused on the visualization of this initial (hours to few days) stages of degradation.

To apply this methodology at a synchrotron beamline is even more challenging. In our case, the combination of a bioreactor-system with a specially developed flow-cell seems to be a promising approach. It could be shown, that the principle application of such a setup is possible, although some limitations occurred: (I) sterility of the system could not be granted over the whole measuring period. This is mainly due to the fact, that hanging rotation stages were used, which give rise to medium contaminations in the chamber. Although a steady flow of argon was applied above the medium, contamination could not be avoided. This fact may be improved by optimizing the flow chambers accordingly to closed systems. (II) Temperature regulation was not constant for the whole system. This was due to heat loss via the tubing. As

Table 1: EDX-analysis of Gd and Nd distribution in the alloy. Sample areas highlighted in Figure 7 C.

\begin{tabular}{ccccccc}
\hline \hline Elt. & \multicolumn{3}{c}{ Concentration [\%] } & \multicolumn{3}{c}{ Atomic [\%] } \\
\hline \hline $\mathrm{Mg}$ & 1 & 2 & 3 & 1 & 2 & 3 \\
$\mathrm{Gd}$ & 84.7 & 69.9 & 91.3 & 97.1 & 93.7 & 98.5 \\
$\mathrm{Nd}$ & 3.1 & 29.0 & 8.3 & 0.5 & 6.0 & 1.4 \\
\hline \hline
\end{tabular}


the degradation of magnesium is temperature dependent ${ }^{6}$, this issue is not negligible when physiological conditions should be applied. Further experiments have shown that a direct heating of the medium in the in situ chamber could solve this problem.

The application of the developed system including flow in this series of measurements revealed, that the total volume of the tube did not change and that the degradation front moved from the outside to the inside of the sample. This is due to the experimental setup, as the flow was applied perpendicular to the sample and gas accumulation inside of the tube was observed. Therefore, no contact of the degradation medium with the inner part of the tube was possible. As only a proof of principle of the functionality of the setup was the aim of the study, this artificial effect can be neglected. However, when working with real prototypes (like stents or bone screws), this behavior is not expected as these are not solid but penetrable structures ${ }^{31,32}$. A further interesting aspect of this behavior is that it is comparable to the behavior in vivo, where the stent structure is still visible after 3-4 months, although the degradation was already finished and the magnesium alloy was completely replaced by calcium phosphates ${ }^{33-35}$.

As an important finding the accumulations of rare earth compounds in the alloy (Gd / Nd), although not individually distinguishable, did not change their position during degradation. This indicates an incorporation in the formed degradation products (most likely calcium phosphates) and to a much lesser extend a release to the environment than theoretically possible. This behavior was observed in other studies ${ }^{36}$ and can be attributed to rare earth elements being known to be "Bone homing" elements in vivo by replacing calcium in the crystal lattice of newly formed bone ${ }^{37-39}$.

\section{CONCLUSION}

This proof of principle study demonstrated the feasibility of a modular in situ closed bioreactor sample environment system for quantitative time-lapse analysis of magnesium degradation under nearly physiological conditions by means of synchrotron based microtomography. The developed experimental setup is a powerful tool to study the principle dynamics and mechanisms of magnesium degradation and for the development and characterization of different newly developed high performance magnesium alloys.

\section{ACKNOWLEDGMENT}

The authors want to acknowledge Bernhard Eltzschig for the manufacturing of the in situ chamber. Nezha Ahmad, Thomas Zander and Zhidan Liu are acknowledged for their help during the beamtimes.

\section{REFERENCES}

[1] ASTM, [G31 - 72(2004) Standard Practice for Laboratory Immersion Corrosion Testing of Metals] ASTM International, West Conshohocken, PA, (2004).

[2] ASTM, [F748-06: Standard Practice for Selecting Generic Biological Test Methods for Materials and Devices] ASTM International, West Conshohocken, PA, (2006).

[3] ISO, [ISO 10993 - Biologische Beurteilung von Medizinprodukten] International Organisation for Standardization, (1999).

[4] F. Witte, N. Hort, C. Vogt et al., "Degradable biomaterials based on magnesium corrosion," Current Opinion in Solid State and Materials Science, 12(5-6), 63-72 (2008).

[5] A. H. Martinez Sanchez, B. J. Luthringer, F. Feyerabend et al., "Mg and Mg alloys: how comparable are in vitro and in vivo corrosion rates? A review,” Acta Biomater, 13(0), 16-31 (2015).

[6] R. Willumeit, F. Feyerabend, and N. Huber, "Magnesium degradation as determined by artificial neural networks,” Acta Biomater, 9(10), 8722-9 (2013).

[7] M. P. Staiger, F. Feyerabend, R. Willumeit et al., "Summary of the panel discussions at the $2^{\text {nd }}$ Symposium on Biodegradable Metals, Maratea, Italy, 2010,” Materials Science and Engineering: B, 176(20), 1596-1599 (2011).

[8] K. Bobe, E. Willbold, I. Morgenthal et al., "In vitro and in vivo evaluation of biodegradable, open-porous scaffolds made of sintered magnesium W4 short fibres,” Acta Biomater, 9(10), 8611-23 (2013).

[9] R. Willumeit, J. Fischer, F. Feyerabend et al., "Chemical surface alteration of biodegradable magnesium exposed to corrosion media,” Acta Biomater, 7(6), 2704-15 (2011).

[10] L. Yang, N. Hort, D. Laipple et al., "Element distribution in the corrosion layer and cytotoxicity of alloy Mg10Dy during in vitro biodegradation,” Acta Biomater, 9(10), 8475-87 (2013). 
[11] L. Yang, N. Hort, R. Willumeit et al., "Effects of corrosion environment and proteins on magnesium corrosion,” Corrosion Engineering, Science and Technology, 47(5), 335-339 (2013).

[12] N. Erdmann, N. Angrisani, J. Reifenrath et al., "Biomechanical testing and degradation analysis of MgCa0.8 alloy screws: A comparative in vivo study in rabbits,” Acta Biomater, 7(3), 1421-1428 (2011).

[13] X. N. Gu, X. H. Xie, N. Li et al., "In vitro and in vivo studies on a Mg-Sr binary alloy system developed as a new kind of biodegradable metal," Acta Biomater, 8(6), 2360-2374 (2012).

[14] F. Witte, J. Fischer, J. Nellesen et al., "In vivo corrosion and corrosion protection of magnesium alloy LAE442,” Acta Biomater, 6(5), 1792-9 (2010).

[15] L. Xu, G. Yu, E. Zhang et al., "In vivo corrosion behavior of Mg-Mn-Zn alloy for bone implant application,” J Biomed Mater Res A, 83(3), 703-11 (2007).

[16] F. Witte, H.-A. Crostack, J. Nellesen et al., [Subtraction-XTM for the Detection of Rare Earth Elements in Magnesium Implants] Hasylab, Hamburg(2003).

[17] F. Witte, F. Feyerabend, P. Maier et al., "Biodegradable magnesium-hydroxyapatite metal matrix composites," Biomaterials, 28(13), 2163-74 (2007).

[18] F. Witte, J. Fischer, J. Nellesen et al., "In vitro and in vivo corrosion measurements of magnesium alloys," Biomaterials, 27(7), 1013-8 (2006).

[19] R. Bernhardt, D. Scharnweber, B. Muller et al., "Comparison of microfocus- and synchrotron X-ray tomography for the analysis of osteointegration around Ti6Al4V implants,” Eur Cell Mater, 7, 42-51; discussion 51 (2004).

[20] R. Bernhardt, J. van den Dolder, S. Bierbaum et al., "Osteoconductive modifications of Ti-implants in a goat defect model: characterization of bone growth with SR $\mu C T$ and histology,” Biomaterials, 26(16), 3009-3019 (2005).

[21] F. Witte, J. Fischer, F. Beckmann et al., "Three-dimensional microstructural analysis of Mg-Al-Zn alloys by synchrotron-radiation-based microtomography,” Scripta Materialia, 58(6), 453-456 (2008).

[22] R. Willumeit, "Neutron and X-Ray Techniques for Biological and Biomaterials Studies,” Advanced Engineering Materials, 13(8), 747-766 (2011).

[23] W. Graeff, L. Bittner, W. Brefeld et al., "HARWI-A hard x-ray wiggler beam at DORIS," Review of Scientific Instruments, 60(7), 1457-1459 (1989).

[24] F. Beckmann, T. Donath, T. Dose et al., "Microtomography using synchrotron radiation at DESY: current status and future developments," Proceedings of SPIE: Developments in X-Ray Tomography IV, 5535, 1-10 (2004).

[25] Q. Peng, Y. Huang, L. Zhou et al., "Preparation and properties of high purity Mg-Y biomaterials," Biomaterials, 31(3), 398-403 (2010).

[26] R. H. Huesman, G. T. Gullberg, W. L. Greenbeg et al., "RECLBL Library Users Manual - Donner Algorithms for Reconstruction Tomography,” (1977).

[27] C. A. Schneider, W. S. Rasband, and K. W. Eliceiri, “NIH Image to ImageJ: 25 years of image analysis,” Nat Methods, 9(7), 671-5 (2012).

[28] A. C. Hanzi, I. Gerber, M. Schinhammer et al., "On the in vitro and in vivo degradation performance and biological response of new biodegradable Mg-Y-Zn alloys,” Acta Biomater, 6(5), 1824-33 (2010).

[29] J. Hofstetter, and E. Martinelli, "Assessing the degradation performance of ultrahigh-purity magnesium in vitro and in vivo," Corrosion Science, 91(0), 29-36 (2015).

[30] N. T. Kirkland, J. Lespagnol, N. Birbilis et al., "A survey of bio-corrosion rates of magnesium alloys,” Corrosion Science, 52(2), 287-291 (2010).

[31] D. E. Allie, “"Magic” Bioabsorbable Metal Stents: The Role of the "Houdini stent” in the disappearance of CLI,” Vascular Disease Managment, 2(4), (2005).

[32] B. Heublein, R. Rohde, V. Kaese et al., "Biocorrosion of magnesium alloys: a new principle in cardiovascular implant technology?,” Heart, 89(6), 651-6 (2003).

[33] T. L. Slottow, R. Pakala, T. Okabe et al., "Optical coherence tomography and intravascular ultrasound imaging of bioabsorbable magnesium stent degradation in porcine coronary arteries," Cardiovasc Revasc Med, 9(4), 248-54 (2008).

[34] R. Waksman, R. Erbel, C. Di Mario et al., "Early- and long-term intravascular ultrasound and angiographic findings after bioabsorbable magnesium stent implantation in human coronary arteries,” JACC Cardiovasc Interv, 2(4), 312-20 (2009).

[35] R. Waksman, R. Pakala, P. K. Kuchulakanti et al., "Safety and efficacy of bioabsorbable magnesium alloy stents in porcine coronary arteries,” Catheter Cardiovasc Interv, 68(4), 607-17; discussion 618-9 (2006). 
[36] N. Ahmad Agha, R. Willumeit-Römer, D. Laipple et al., "The Degradation Interface of Magnesium Based Alloys in Direct Contact with Human Primary Osteoblast Cells,” PLoS One, 11(6), e0157874 (2016).

[37] S. Zaichick, and V. Zaichick, "Human bone as a biological material for environmental monitoring," International Journal of Environment and Health, 4(2/3), 278-292 (2010).

[38] S. Zaichick, V. Zaichick, V. Karandashev et al., "Accumulation of rare earth elements in human bone within the lifespan,” Metallomics, 3(2), 186-194 (2011).

[39] V. Zaichick, "Chemical elements of human bone tissue investigated by nuclear analytical and related methods," Biol Trace Elem Res, 153(1-3), 84-99 (2013). 